A Multi-Case Investigation of Electronic Health Record Implementation in Small- and Medium-Size Physician Practices

By: Steven Wallace, Keith Maxey, and Lakshmi S. Iyer.

Wallace, S., S., Maxey, K. \& Iyer, L.S. (2014). "A Multicase Investigation of Electronic Health Record (EHR) Implementation in Small and Medium Size Physician Practices," Journal of Information Technology Case and Application Research. Vol 16, No. 1, 27-48.

***@) Taylor \& Francis. Reprinted with permission. No further reproduction is authorized without written permission from Taylor \& Francis. This version of the document is not the version of record. Figures and/or pictures may be missing from this format of the document. $* * *$

This is an Accepted Manuscript of an article published by Taylor \& Francis in Journal of Information Technology Case and Application Research on 06/09/2014, available online: http://www.tandfonline.com/10.1080/15228053.2014.912478

\begin{abstract}
:
The American healthcare system has undergone significant changes as health information technology (HIT) has evolved. According to the Institute of Medicine (IOM, 2001), "Automation of clinical, financial, and administrative transactions is essential to improving quality, preventing errors, enhancing consumer confidence in the health system, and improving efficiency" (p. 16). HIT helps target improvements in patient care through improved adherence to clinical guidelines, improved monitoring of diseases, reduction in medical and medication errors, and access to more accurate and complete health information that aids in the decision-making process (Cecchini, 2011a). However, these improvements are not guaranteed just because an electronic health record (EHR) is implemented. Improvement in the quality of care is dependent on what the specific EHR offers in the way of functionality and how it affects providers and office staff (Carayon, Smith, Hundt, Kuruchittham, \& Li 2009).
\end{abstract}

Keywords: American healthcare system | health information technology (HIT) | electronic healthcare record (EHR)

\title{
Article:
}

The American healthcare system has undergone significant changes as health information technology (HIT) has evolved. According to the Institute of Medicine (IOM, 2001), "Automation of clinical, financial, and administrative transactions is essential to improving quality, preventing errors, enhancing consumer confidence in the health system, and improving efficiency" (p. 16). HIT helps target improvements in patient care through improved adherence to clinical guidelines, improved monitoring of diseases, reduction in medical and medication errors, and access to more accurate and complete health information that aids in the decision-making process (Cecchini, 2011a). However, these improvements are not guaranteed just because an electronic health record (EHR) is implemented. Improvement in the quality of care is dependent 
on what the specific EHR offers in the way of functionality and how it affects providers and office staff (Carayon, Smith, Hundt, Kuruchittham, \& Li 2009).

As part of the American Recovery and Reinvestment Act (ARRA), the Health Information Technology Economic and Clinical Health (HITECH) Act was created to incentivize the use of HIT programs (Henricks, 2011). As of July 2013, \$15.5 billion was awarded for Medicare and Medicaid providers that achieved Centers for Medicare and Medicaid Services (CMS) metrics on meaningful use (MU) measures (CMS, 2014). The HITECH Act aims to improve both patient health and the functioning of health systems through MU of health technology (HRSA, 2013).

Although there have been multiple studies on HIT impact on large hospitals, few studies have focused on EHRs within small-and medium-size physician practices (SMPPs). Unlike their larger counterparts, SMPPs have several unique challenges with HIT implementation (Reardon \& Davidson, 2007). Unless SMPPs are subsidized, HIT systems can be too expensive to implement. SMPPs are also ill equipped to deal with the lost revenue that is incurred during implementation due to lost productivity. In a literature review on EMR implementation, Ludwick and Doucette (2009) stated, "few studies examined individual or small (less than three physicians) practitioner experiences" (p. 27). In a 2011 study, Reardon claimed that there was little to no theory-based HIT studies at the small physician practice level. SMPPs are medical practices that employ five to 20 physicians (Gans et al., 2005; Lorenzi et al., 2009). These practices are broken down into two categories, independent physician practices (IPPs) and affiliated physician practices (APPs) (Beasley et al., 2005; Isaacs et al., 2009; Tollen, 2008). IPPs are owned and operated by the attending physicians while APPs are contracted by a larger medical organization such as a hospital or managed care group.

The goal of this study is to examine the implementation of EHRs within SMPPs and provide recommendations on how to successfully implement an EHR system within a small practice. The second goal of this study is to examine the impact of EHR implementation on quality within the SMPP. We use Marcus and Tannis (2000) process theory to examine prior work in EHR implementation and use that framework as a foundation for our case studies. The findings of this study will provide a better understanding of how these small organizations tackle the challenges of implementing a large project. It will also provide insight on the best practices with regard to the process of EHR implementation and offer practitioners some guidelines as to what works best in SMPPs.

In the next section, we provide an overview of EHRs, EHR implementation stages, and a summary of prior research into SMPPs. Then we discuss the methodology, including a brief summary of the practices, questions asked, and factors used to analyze the EHR impact on quality. Next we discuss our findings and overall themes taken from the data. Finally, we offer recommendations for SMPPs that intend to implement an EHR system, followed by the study conclusion.

\section{EHR System}

An EHR system is software that can support the following capabilities: store and retrieve patient clinical and demographic information; access and update laboratory tests and results; order entry, 
including pharmaceutical prescriptions; support clinical decisions, including drug interactions (Eastaugh, 2012). Venkatraman Bala, Venkatesh, and Bates (2008) defined anEMR as an "automated clinical system that generally includes data related to medical history, patient demographics, clinician's notes, drug information, electronic proscriptions and diagnostic test orders" (p. 140). In theory, EMRs are designed to follow a patient with no regard for location (Dey, Sinha, \& Thirumalai, 2013; Williams \& Boren, 2008;). For example, the primary care physician and any number of specialists can review a patient's EMR even if these physicians are located in different states. In practice, there are no standards with these records because each application is tailored for individual practices; therefore, it is difficult to transfer records between offices with applications from different venders (Hoffman, 2009; Venkatraman et al.,2008). In some hospitals, different departments utilize EMRs from different venders (Venkatraman et al., 2008).

Hoffman (2009) noted other challenges to EMRs in addition to the issue of standardization. The first is the challenge of adoption. While most physician offices will front the cost of the system, they will not receive the majority of the benefits of adoption. Instead, insurance companies benefit through cost savings such as a reduction in duplicated tests for individual patients. Another challenge to EMRs is complying with the federal Health Insurance Portability and Accountability Act of 1996 (HIPAA) statutes, and different state regulations surrounding medical privacy. Garret and Seidman (2011) argue that the difference between EMRs and EHRs is that EHRs are accessible by all healthcare providers regardless of locale. Across the literature,EMRs are synonymous with EHRs and defined as capable of distributing electronic records across locations (Dey et al., 2013; Williams \& Boren, 2008). For the purposes of this study, we consider EMRs as EHRs.

According to Columbus (2006), the purchase of an EHR system is among the most significant decisions that a small medical practice will make due to the large investment of both time and money. For a single physician practice, implementation time can range from 12 to 18 months. As the number of physicians increases, so does the time needed for implementation. A study conducted by the Agency for Healthcare Research and Quality (AHRQ) of 26 primary care practices looked at the time required for planning and implementation (Fleming et al., 2011). The study found that an average practice with five physicians took 611 hours for planning and implementation, or 134 hours per physician. This length of time includes, for example, analysis or assessment of pre-EHR conditions and workflow, vision and goal setting for desired outcomes, EHR comparison and selection, training, implementation, and evaluation.

\section{Information Technology (IT) Implementation Theories}

Since this study focuses on EMR implementation and not adoption, we first examined prior work in the information science (IS) literature on IT/IS implementations. Koh, Soh, and Markus (2000) used a process model developed by Markus and Tanis (2000) to map the implementation of an ERP. Markus and Tanis (2000) argued that goals will not always be achieved even if a list of necessary conditions is met as other factors may derail the implementation. The necessary conditions include user involvement, IT skills of users and IT staff, management commitment, and user expectations. They categorized the implementation process into four phases: chartering phase, project phase, shakedown phase, and onward and upward phase. The outcomes of each 
phase will become the condition for starting the next phase. Koh et al. (2000) found that the project phase in their case study was overly long because of a lack of local IT staff. The organization had to rely on remote support, which delayed the implementation. They were also hampered in the shakedown phase due to the difficulties of the customization of the software. They also observed many disruptions due to a lack of focus on training. They argued that if the organization had placed more emphasis on training, they would have seen fewer problems during implementation. We use this process theory as a framework to examine how EMR systems are implemented.

In order to gain understanding in the implementation of an order management system (OMS), Joshi (2005) developed the equity implementation model. The author explained that people behave rationally in their own best interest and so change is not automatically rejected. For example, people do not usually object to pay raises or promotions. When a change occurs, a person will evaluate that change and will only become resistant if they find the change is unfavorable. The author argues that favorability is mostly based on a sense of equity. For example, when a new system is implemented, will the user be treated equally compared with other users? Joshi's examination of the OMS system found that users were resistant to the change because of a drop in productivity and that did not compare themselves against other users or their employer. We will use this theory to explain why some users are resistant to the introduction of technology that they perceive as disruptive.

\section{EHR Implementation}

Based on our literature, we did not find any explicit use of process theory for EHR implementation. We use the process theory by Marcus and Tannis (2000) as a framework to examine prior work in EHR system implementation. The following subsections give a brief summary of the elements of the process theory (Figure 1).

FIGURE 1 Electronic health record (EHR) implementation and use process. Source: Markus and Tanis (2000, p. 189).

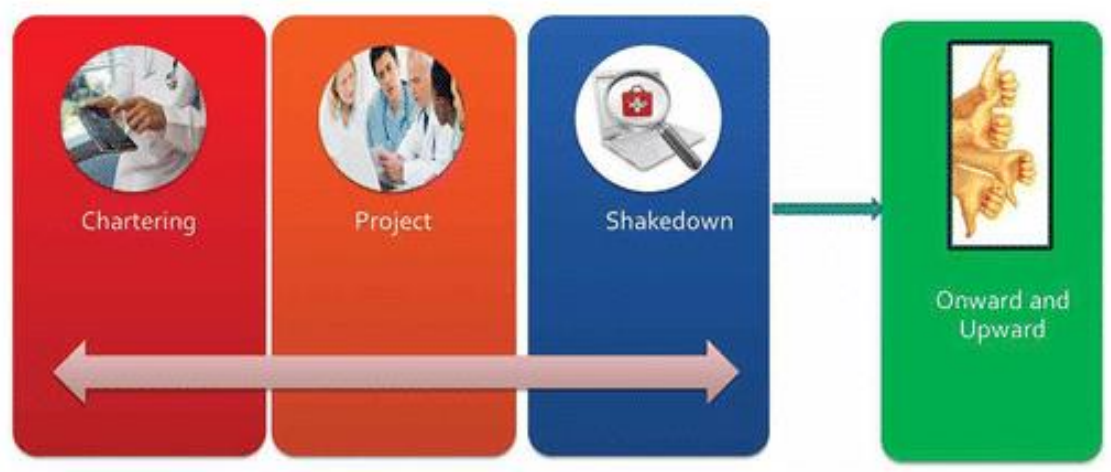

\section{Chartering}

The chartering phase is when the organization sets its goals and lists of system requirements. The chartering phase is similar to the combination of the planning and analysis phase in the Systems Development Life Cycle (SDLC) model. Before implementing an EHR, a medical 
practice must carefully analyze several items: user technical proficiency, current state of IT infrastructure, current workflows, and any requirements specific for that particular practice. The Office of the National Coordinator for Health Information Technology (ONC) also recommends that a practice sets specific goals that the EHR system implementation should achieve (HealthIT, 2013). These goals will help focus the implementation team to ensure that the project does not exceed its scope. Finally, the ONC recommends that a transition plan should be created to move existing records and data from the current system to the new EHR to ensure that no data are lost. This is the phase in which money is allocated for the next phase, the project phase (Marcus \& Tannis, 2000).

\section{Project}

The project phase is where the system is implemented within the organization. Marcus and Tannis (2000) state that this is the phase in which the cash allocated from the prior phase is converted into assets. The first step in this phase is to use the data collected from the chartering phase to select an appropriate EHR system (HealthIT, 2013; Marcus \& Tannis, 2000). A 2013Black Book Rankings survey of almost 17,000 physicians found that nearly 50\% were not satisfied with their EHRs (Wietecha,2013). Another recent survey from AmericanEHR Partners of more 4,000 physicians found a trend of increasing dissatisfaction with EHR performance and usability. (Terry, 2013) These statistics help emphasize the utmost care that is needed in EHR selection. Some criteria to consider when selecting an EHR system are system certification, vendor reputation, features included, level of IT support, and the scalability of the system (Cecchini, 2011b).

The next step is the actual implementation of the EHR system. This involves pilot testing, practice go-live events, and the go-live date. A pilot test is necessary to ensure that "data tables and files have been loaded properly, data collected are processed and stored correctly, interfaces work, workflows have been adjusted appropriately, alerts fire correctly, and reports are able to be generated accurately and completely" (National Learning Consortium [NLC], 2012a, p. 2). It is recommended that the vendor be involved with the testing, but the testing should be done in conjunction with someone from the practice. This will give the vendor a chance to address any issues that occur during the pilot. After the pilot run, then the site can then implement the rest of the system at the go-live date. After the go-live has occurred, the Shakedown phase can occur.

\section{Shakedown}

The shakedown phase is where any problems in implementation are addressed and the system is adapted to the organization. Once the system is running without any problems, the practice then has an opportunity to get the system customized by the vendor (NLC, 2012a). This phase is also where the users are trained to use the new system. According to Jason Mitchell, who directs the Center for Health Information Technology at the American Academy of Family Physicians (Leawood, KS), "I have not been made aware of any EHR implementation program that failed because of too much training, but I know of a number that have occurred because of too little" (Downing Peck, 2013, para. 3). 
The training provided should not only cover the goals that were determined in the chartering phase, but also MU compliance and privacy and security issues for HIPAA compliance. Data migration should also occur during this stage (HealthIT, 2013). Concentrating on specific quality measures, it was determined that practices required "a minimum of nine months of EHR exposure, combined with eight or more technical assistance visits, to demonstrate any significant statistical improvements" (Manos, 2013, para. 6). This study found that practices with little or no technical help failed to demonstrate any statistical improvements, even after using the EHR for 2 years.

\section{Onward and Upward}

The onward and upward phase occurs when the system is finally stabilized. During this final phase, maintenance is performed and upgrades are implemented. In addition, ongoing evaluations are needed to ensure that the goals are met, efficiency and patient care are improved, and both patient and staff satisfaction are present. It is also recommended that workflow effectiveness be evaluated and adjustments made. Finally, SMPPs may benefit from continuous quality improvement (CQI) after EHR implementation. In health care, this means that staff continue to ask "How are we doing?" and "Can we do it better"? (Edwards, 2008) CQI strategies can help a practice achieve MU, maximize the benefits of EHR, and improve the quality of care. CQI will help to ensure efficient workflows and few workarounds (NLC, 2012b).

\section{SMPPs With EHRs}

In this section, we review prior literature on SMPPs' experience with EHRs and HITs and highlight the research focus, methodology, and key findings of the studies in Table 1. In a literature review on EMR implementation, Ludwick and Doucette (2009) stated, "few studies examined individual or small (less than three physicians) practitioner experiences" (p. 27). In a 2011 study, Reardon claimed that there were little to no theory-based HIT studies at the small physician practice level. 
TABLE 1

Summary of Studies on Small- and Medium-Size Physician Practices (SMPPs) in Context of Electronic Health Records (EHRs) and Health Information Technology (HIT)

\begin{tabular}{|c|c|c|}
\hline Authors & $\begin{array}{c}\text { Research } \\
\text { Focus/Methodology }\end{array}$ & Findings \\
\hline Gans et al. (2005) & EHR Adoption/Survey & $\begin{array}{l}\text { - SMPPs were less likely than large practices to have or plan } \\
\text { to have EHRs } \\
\text { - Initial reduction of productivity, but users believed that } \\
\text { revenues would eventually increase }\end{array}$ \\
\hline Baron et al. (2005) & $\begin{array}{l}\text { EHR } \\
\text { Implementation/Single } \\
\text { Case Study }\end{array}$ & $\begin{array}{l}\text { - EHR had a negative impact financially as more time was } \\
\text { spent on system interface with the system and less patients } \\
\text { seen per day } \\
\text { - Due to disruption of the implementation phase, customer } \\
\text { service was negatively impacted during that time period } \\
\text { - Practices had to redesign every workflow in to } \\
\text { accommodate the new EHR }\end{array}$ \\
\hline $\begin{array}{l}\text { Davidson and } \\
\text { Helsinga (2007) }\end{array}$ & $\begin{array}{l}\text { EHR Adoption/Action } \\
\text { Rescarch }\end{array}$ & $\begin{array}{l}\text { Barriers to adoption: } \\
\text { - Too costly to install } \\
\text { - Integration difficulty both internally and externally }\end{array}$ \\
\hline $\begin{array}{l}\text { Reardon and } \\
\text { Davidson } \\
\text { (2007) }\end{array}$ & $\begin{array}{l}\text { EMR } \\
\text { Implementation/Survey }\end{array}$ & $\begin{array}{l}\text { Growing practices and practices that already used IT had } \\
\text { higher rates of assimilation success }\end{array}$ \\
\hline $\begin{array}{l}\text { Lorenzi et al. } \\
\quad(2009)\end{array}$ & $\begin{array}{l}\text { EHR Benefits and } \\
\text { Barriers/Overview }\end{array}$ & $\begin{array}{l}\text { EHRs benefit small practices by: } \\
\text { - Improve patient care through quick communication between } \\
\text { staff and provide quick and easy access to patient records } \\
\text { - Improve office efficiency by reducing paper work and time } \\
\text { to search for relevant patient information. } \\
\text { - Provide financial gains through staff reduction such as } \\
\text { records transcribers; can potentially increase number of } \\
\text { patients/day } \\
\text { EHR barriers include: } \\
\text { - No set of standards to dictate how records are stored } \\
\text { - Steep learning curve; small practices have fewer resources } \\
\text { available to provide training opportunities }\end{array}$ \\
\hline $\begin{array}{l}\text { O'Neill et al. }^{\prime} \\
\text { (2011) }\end{array}$ & EMR Adoption/Survey & $\begin{array}{l}\text { - } 28 \% \text { had adopted an EMR } \\
\text { - } 14 \% \text { had fully implemented EMR } \\
\text { - Younger physicians more likely to adopt a system } \\
\text { - EMR adoption was significantly higher in rural areas than } \\
\text { their urban practices }\end{array}$ \\
\hline $\begin{array}{l}\text { Bardach et al. } \\
\text { (2013) }\end{array}$ & $\begin{array}{l}\text { EHR } \\
\text { Impact/Experimental }\end{array}$ & $\begin{array}{l}\text { The group that was incentivized performed significantly } \\
\text { better than the control group in all categories except in } \\
\text { cholesterol numbers }\end{array}$ \\
\hline
\end{tabular}

Prior SMPP studies with EHRs can be classified into two categories: EHR adoption and EHR implementation. The first category is the study of challenges surrounding EHR adoption. Gans et al. (2005) conducted a survey to study the rates of EHR adoption based on practice sizes. Davidson and Helsinga (2007) used action research to study EHR adoption in small physician practices in Hawaii. This study covered the outreach phase of recruiting physician practices to adopt EHR and covered 26 site visits that included interviews with physicians. O'Neill et al. (2011) surveyed 482 physicians to measure the adoption rate of EMRs in small practices in Kentucky.

While the previous studies focused on EHR adoption, other studies in our review focused on the actual EHR implementation. Baron et al. (2005) conducted a single case study of the impact of the implementation of the EHRs on their small practice. Reardon and Davidson (2007) conducted a survey in Hawaii to study the assimilation of EMRs in small physician practices. In 
their survey of 130 practices, 90\% had three or fewer physicians on staff. Bardach et al. (2013) examined the effects of pay-for-performance (P4P) on quality of care in small practices that use EHRs. Lorenzi et al. (2009) provided an overview of EHRs used in small ambulatory practices, which they defined as practices with five or less physicians.

Unlike prior studies, this research uses a qualitative approach to examine the implementation process within SMPPs and how the implementation has impacted those practices in various ways. By examining multiple cases qualitatively, we can gain a better understanding of how EHR implementation processes are performed in small healthcare settings. In the next section we explain how cases were selected and how the data were collected.

\section{METHODOLOGY}

We use the case study methodology outlined by Yin (2009) in this research. We take an interpretive approach to this study, and thus, unlike the positivist approach, no pre-determined dependent and independent variables are used. Instead, we are searching for a richer understanding of the phenomenon under study (Klein \& Myers, 1999). The theories described in the previous section are used to guide the research design in order to gain appropriate insights for data analysis (Walsham,2006). Research has shown that case studies provide a deep understanding in both the healthcare context and in IS implementation. Both Palvia and Thambusamy (2013) and Kealy and Stapleton (2011) have shown that case study is an excellent method for providing a deep look at the healthcare sector. Other research has shown that the case study also provides a better understanding of IS implementation: IT infrastructure library implementation (Pederson Kraemmergaard, Lynge, \& Schou, 2010); ERP implementation (Furumo \& Melcher, 2006; Koh et al., 2000); order management system (Joshi,2005); and, EHR/EMR implementation (Baron et al., 2005).

\section{Site Selection and Case Summary}

We limited our site selection to those SMPPs that are currently using EHRs and that have no more than 20 physicians working in each practice. We chose practices that have either finished EHR implementation or are in the process of implementation. We contacted sseveral SMPPs that met the selection criterion, and found five practices that were willing to participate in the study. Given that qualitative studies demand time commitment of the participating organizations, we anticipated that our request for research participation would result in a smaller response rate as most small practices have significant demands on their time because they are not heavily staffed. For this reason, we were only able to interview a single key informant from the first four cases. In each of those cases, the key informant was a physician. We were able to interview more users in the fifth case. Those users included a physician, a nursing supervisor, two front-office staff members, and the office administrator. The semi-structured interviews averaged 45 minutes per interview. The next section gives a sample of the initial interview questions.

Table 2 provides a brief summary each practice. In the first three cases, the SMPP used the same EHR, while the Practice 4 and 5 used a separate EHR. All of the practices except Practice 5 were APPs associated with a parent health organization. Each SMPP is located in the southeastern region of the United States. Data from the first four cases were gathered in the fall of 2013. Data 
from Practice 5 were gathered in fall and winter of 2010/2011 in the study performed by Albert, Wallace, and Iyer (2011).

TABLE 2

Summary of Practice Types, Time Span of Electronic Health Record (EHR) Use, and Number of Physicians for the Study Small- and Medium-Size Physician Practices (SMPPs)

\begin{tabular}{llcr}
\hline Case & \multicolumn{1}{c}{ Type } & Time Span of EHR Use & Number of Physicians \\
\hline Practice 1 & Primary care, affiliated physician practice (APP) & 2 years & 4 \\
Practice 2 & Primary care, APP & 1.5 years & 1 \\
Practice 3 & Primary care office, APP & 1 year & 12 \\
Practice 4 & Pediatrics, APP & 5 years & 11 \\
Practice 5 & Primary care, independent physician practice (IPP) & $<1$ year & 8 \\
\hline
\end{tabular}

\section{Interview Questions}

In addition to demographics questions, a sample of the questions used in each of the interviews related to EHR implementation and use follow:

- How long has the office used the current EHR?

- Was the current EHR implemented strictly for compliance with meaningful use and HIPAA or for additional goals? If it was for additional goals, what goals were set forth to accomplish? Do you think the goals were met?

- What specific role, if any, did you play with implementation?

- What are the most important considerations to keep in mind for EHR selection?

- What are the most important pre-implementation tasks to complete?

- What "workarounds" were necessary, if any?

- How much pre-implementation training did you receive?

- What are some of the most challenging aspects of EHR rollout?

- How long did it take until you think efficiency returned to or even surpassed pre-EHR levels?

- Do you think that patient care has improved?

- What would you do differently if you went through another implementation?

- How many patients per day do you see? How many at "go-live"?

- What is the definition of "quality care" as viewed by the practice?

- As a whole, how does the practice define safety, effectiveness, timeliness, patient centeredness and efficiency?

\section{Factors to Measure Quality}

To measure the impact of the new EHRs, we used Berwick's (2002) measurements of quality: safety, effectiveness, patient-centeredness, timeliness, efficiency, and equity. This study is inclusive of all elements of the quality except equity. Equity in patient care refers to closing the gaps in racial and ethnic disparities within health care. Addressing equity is done on the part of health care professionals to provide care to individuals and to determine what level that care will be administered. These issues are outside of the scope of this study because an EHR cannot affect business processes that impact equity in patient care. Table 3 presents the base definitions of the five aspects of the quality chasm as addressed in this study. 
TABLE 3

Definitions of Quality Elements

\begin{tabular}{ll}
\hline Element & \multicolumn{1}{c}{ Definition } \\
\hline Safety & Reduction of errors by all stakeholders \\
Effectiveness & Correlation of health system to science \\
Patient-Centeredness & Respect for patient and their decisions regarding personal health \\
Timeliness & Reduction in time spent waiting on and administering patient care \\
Efficiency & Reduction in total cost of care \\
\hline
\end{tabular}

Source: Berwick (2002).

\section{Safety}

Gaal, Laarhoven, Wolters, Wetzels, Verstappen, and Wensing (2010) define safety from a practitioner point of view: Ensure that no harm is done to the patient. This study defines patient safety as the reduction of errors by all stakeholders. Some of the factors in patient safety are medication safety, communication between practitioners and between practitioners and patients, incident reporting, patient responsibilities, and medical knowledge.

\section{Effectiveness}

In this study, effectiveness is defined as how well the EHR is correlated to science (Berwick, 2002). Berwick (2002) proposes several ways in which effectiveness can be enhanced. The first way is to allow the patient to receive care outside the office visits through channels such as Internet or phone. Another way is for the care to be customized for the patient and to give the patient control. Other areas for improved effectiveness include transparency, evidence-based decisions, anticipated needs, and cooperation between the caregivers. Some factors related to effectiveness are system quality, information quality, service quality, frequency of use, and user satisfaction (Otieno, Hinako, Motohiro, Daisuke, \& Keiko, 2008).

\section{Patient-Centeredness}

Berwick (2002) defines patient-centeredness as "health care should honor the individual patient, respecting the patient's choices, culture, social context, and specific needs" (para. 10). Robinson, Callister, Berry, and Dearing (2008) documented four different views of patientcenteredness: public policy perspective, economic perspective, clinical practice perspective, and $a$ patient perspective. This study will focus on the patient perspective and define patientcenteredness as when decisions are based on what the patient wants or needs and the patients are given the information to make those decisions.

\section{Timeliness}

Timeliness can be defined as "receiving care when needed" (Berry, Seiders, \& Wilder, 2003, p. 568). This term refers to coordinating the times for the patient to see the primary care and specialty physicians (Berry et al., 2003; Berwick, 2002). This can also include the time during which the patient is waiting for a response from the physician either by phone or e-mail (Couchman, Forjuoh, \& Rascoe, 2001).

\section{Efficiency}


In this study, efficiency is defined as the reduction in health care costs (Berwick, 2002;

Thompson \& Dean, 2009;). This factor can be accomplished with reduction in waste (Bentley Effros, Palar, \& Keeler, 2008; Berwick, 2002) or by reducing the amount of time needed to record data (Ward, Stevens, Brentnall, \& Briddon, 2008). Waste can take the form of redundant processes or forms, unnecessary procedures, or bad management of personnel time.

\section{FINDINGS}

The following are the main themes that were extracted from the interviews.

\section{Implementation Process Model}

Using the Marcus and Tannis (2000) implementation process model (IPM), we were able to chart the progress of the EHR implementation in these five cases. Because we did not gather any data prior to the project phase, we do not have any information for the chartering phase in any of these cases. One large difference found in the project phase was between the first four practices and Practice 5. Only in Practice 5 was the implementation performed in staggered steps with only a few physicians switching to the new system at the beginning and the other physicians slowly transitioning after the initial issues were resolved. In none of the cases was a pilot run actually performed.

The IPM called for the training to be offered after the system had been completely implemented. In every case, training was offered prior to implementation. Implementation practices may have changed since IPM was first advocated, hence the difference. It may also be due to the fact that these SMPPs do not have the luxury to have the time to train users while performing their normal duties.

\section{Lack of Common Language}

During some of the interviews, it became apparent that the physicians were not well versed in business and technology terminology. For two of the physicians, it was necessary to explain the concepts of workflows and technology workarounds. Because most of these practices were APPs that were affiliated with larger organizations and did not have to personally supervise the implementation, they were not properly educated in EHR implementation procedures. Practice 5, an IPP, may have been better versed in the terminology because of a greater stake in the new system.

\section{Training}

One of the biggest themes from the case data is the importance of training for the new EHR system. Each subject emphasized the need for proper training before the site fully implements the new system. Practice 4 complained that the 1 week of training was not enough and more training would have been better. Practice 1 complained that evening trainings were not effective after working a full shift. 
Some events were offered after hours - not effective after working an entire day. (Primary care physician from Practice 1)

Practice 2 reported several of 8-hour training sessions that included a sandbox mode ${ }^{1}$ for experimenting with the new features.

We had two or three eight hour sessions. There was also a playground available. (Primary care physician from Practice 2)

\section{Need for Simple User Interface}

In most of the cases, the subject stated that it was important that the EHR system selected would be simple to use. Practice 1 and 3 mentioned that a small number of screen changes when completing a medical chart:

Make sure the EHR isn't over-complicated. Make sure there aren't too many screen changes needed to complete a chart. (Primary care physician from Practice 3)

What took two clicks with the old EHR now takes fifteen clicks with the new EHR. (Primary care physician from Practice 1)

Practice 2 wanted the system to be customizable by the user and to provide dashboard support for easy monitoring. Prior to the implementation, the system for Practice 2 was changed to allow their notes to be visible on one-half of the screen at all times to prevent the need for frequent switching of screens.

\section{Initial Reduction in Productivity}

All of the practices had a reduction in patient load at the beginning of the system rollout. There were some differences in the extent of reduction and the amount of time until they were back at a full patient load. Practice 1 complained that patient examinations still takes longer postimplementation.

The workflow did not change any with [the new system], but it took, and still takes, a lot longer. (Primary care physician from Practice 1)

Practice 2 was able to reach normal workload levels after 1 month and saw improvements to preimplementation levels after 3 months. They also stated that billing became more efficient with the new system. After implementation, Practice 5 saw increased efficiency with regard to record retrieval and filling prescriptions.

\section{The Successful Rollout}

One major difference was noted between the practices during the rollout. Two of the practices (Practices 1 and 3) had a high level of frustration during the rollout period of the EHR. In contrast, Practices 2 and 5) were from a different health system and had a more positive view of 
the rollout experience. As mentioned previously, Practice 2 found better efficiency with an increase number of claims that processed with no errors. Practice 5 had quicker record retrieval and proscription fulfillment. Practice 5 also saw improvements in quality of care due to the use of templates. System templates forced users to ask questions that might have been missed prior to system implementation.

In addition to more training, Practices 2 and 5 also staged the implementation into two parts: practice management and clinical. In both cases, the practice management portion of the system was implemented 6 months prior to the implementation of the clinical portion. This allowed better continuity as the practice management portion was completely implemented before the clinical portion began. This had the added benefit of halving the potential issues at time of implementation by dividing the system in two. The physician at Practice 2 was also enthusiastic about the new EHR system, which could have led to the physician undergoing more training classes and their encouragement of the staff to take more training. In addition to separating the rollout of the EHR, Practice 5 also staggered the rollout across the physicians by having each physician switch over to the new system one at a time. This process took a total of 2 months. This allowed issues to be resolved with the first physician before the other physicians had to make the switch.

\section{Impact on Quality}

We were able to do a more thorough study of Practice 5, which allowed us to measure its EHR's impact on quality. In the following sections we discuss how the EHR impacted the different elements of quality.

\section{Safety}

This EHR system has improved safety in Practice 5 in several ways. The system prevents the wrong chart from being retrieved for a patient. With certain demographic information required by MU, it is more difficult to retrieve the wrong patient data in front of a clinical staff member.

The use of typed data has mitigated the dangers of misinterpreting a physician's handwriting. This point was vital when a physician was examining another physician's patient. Before the EHR was implemented, if a physician could not read the notes, then the physician would have to start the patient investigation as if the new patient were new. Any prior knowledge would be lost, and some vital information could have been overlooked. Now, the physicians have access to legible notes inside and outside the practice. The electronic charts are better organized than paper copies, which helps prevent a physician from overlooking something vital. With electronically submitted prescriptions, the pharmacy no longer has to rely on deciphering the physician's handwriting, which leads to more accurately filled prescriptions.

Drug interactions are displayed in EHR based on the patient's prescriptions and alerts the staff to possible problems with the current mix of drugs. Then the physician must decide if the benefits outweigh the negatives. In addition to medications, allergy alerts also appear with a flag or alert. When asked about this, feature primary care physician from Practice 2 stated that the new system had: "some awesome dashboards for analytics" (Primary care physician from Practice 2). 
The EHR also improves safety by giving the physician a better overview of the patient's health history and allows the physician to see what drug was prescribed and when. This feature will help remind the physician of any follow-ups needed while the patient is in the office. Finally, the system forces the physician to document certain information that forces the physician to ask why a patient was not given a particular treatment or prescription. This event could be due to a finding at the last visit or a note lost in a paper chart that contains several loose pieces of paper.

The EHR allows the practice to review the treatments and prescriptions that the patients are currently taking and allows the practice to consult with the patient about the best insurance plan to choose in order to cover all of the patient's needs. This approach is especially true for Medicare patients who are bombarded with information and telemarketers and who look to the practice to guide their decisions. The new system helps the practice educate patients on the plan.

\section{Patient-Centeredness}

The EHR-provided patient alerts can be seen as patient centered. In one example, the system will alert the practice if a patient needs a wheelchair, even if they do not come in with one. If patient is blind or hearing impaired, the EHR will alert the practice that the patient has additional needs. In another example, the system alerts the practice who to contact in cases in which the patient is unable to comprehend the health information. In the past, the staff member may not be aware of the situation and call the patient instead of the contact.

The EHR also has functionality to ensure confidentiality. Charts can be marked "sensitive" and security has to be assigned to those individuals who are allowed to access those charts. In addition, the staff is aware that the director can look at who is accessing what chart and why. This knowledge acts as a control because there is a permanent record who entered what data and who accessed it.

The EHR also negatively impacts patient-centeredness in a few ways. The system does not customize at the patient level and forces the clinical staff to treat all patients the same way in the form of questions that it asks. The data entry aspect of the system also takes away from the "face time" with the physician. The laptop could also become a barrier between the physician and the patient and, in some cases, may prevent the patient from sharing health information because of privacy fears.

One way to mitigate those disadvantages was found when one physician (in Practice 5) did not enter all of the information at the time of the visit. Instead, he waits until after office hours to finish the documentation of all his patients. This takes more time and can take the physician an additional 2 hours to complete.

The current EHR does not handle waivers very well. The patients do not have access to a patient portal where they can electronically sign waivers for HIPAA and Medicare, so they must sign a hard copy. Those waivers are not scanned and not kept, but they are noted within the system. When the patient portal is implemented, the practice hopes to include electronic consents and waivers. 


\section{Timeliness}

The EHR has sped up many practice processes, including electronic prescribing, billing, and filing insurance. Clinical staff members no longer have to search for the physical location for a paper chart, which reduces the time that a patient has to wait on the phone for information. The EHR also enables faster access to the physicians. When there is a question for the physician, a flag is sent to the desktop computer for the physician, who can respond quickly. The staff no longer has to try to translate physician handwriting, which improves their timeliness. The staff has access to the physician's notes for patients, which allows them to give the same information to patients that the physician gave the patients earlier without the staff having to take time to consult with the physician.

The front office staff is better able to watch the accuracy of charges due to their improved access to the charts. If they have questions, they have the ability to consult the electronic chart without having to search for a paper chart. Digital signatures are now being accepted with the EHR. This has improved accuracy because signatures on claims are legible and improves the speed in which claims are processed. With charges entered on one day and submitted the next, the payments are now arriving more quickly. With this EHR, the front office staff can send multiple claims in one batch, which allows them to process at a quicker rate. The system allows the staff to preview the checks that have been approved and to know what payments are coming in from Medicare and the health insurance companies. Reports are also more easily generated and accessed.

Practice 1 had a different outcome regarding the EHR impact on timeliness. They are still not examining as many patients compared to pre-implementation levels:

Still not quite there. It is hard to see the same number of patients on a busy day as pre-EHR. (Primary care physician from Practice 1)

\section{Efficiency}

There have been improvements in efficiency with regards to retrieving information. The EHR allows the user to retrieve a patient's electronic chart quickly to immediately retrieve the information they want. This information can be retrieved from within the practice and from outside the practice if the clinical staff has access to an Internet connection. Unfortunately, the local hospital does not allow Internet access within their ER.

The EHR has also improved efficiency in other situations. It has decreased the time to write prescriptions since they can now be filled electronically. Ordering laboratory tests is also easier with the new system. Physicians can now use the system to order a patient's tests, and the communication between the laboratory and physician has been reduced from a phone call to a text message. With regard to the nurses' time, the system will decrease the time spent with calls. As mentioned previously, retrieving patient's electronic charts take less time now, which decreases the time spent handling patient phone calls and requests. The need for office supplies has also decreased since specialized paper forms are no longer needed. Finally, the practice no longer uses their fax machine, so that change has freed some office space and reduced a small 
amount of electrical use. When asked about efficiency, Practice 2 observed improved efficiencies in their practice:

Billing has gotten incredibly more efficient as well as coding and number of clean claims. (Primary care physician from Practice 2)

Since the data entry takes more time than before, the EHR has decreased efficiency on that end of the process. As mentioned previously, the physicians are taking more time to write their visit reports and it takes time to convert the paper charts to electronic charts before each visit. As the physicians become more used to the new system, they might become more efficient at data entry. The biggest savings will occur when all of the patient information is finally entered into the new EHR, which will allow the practice to reduce their labor costs in the form of chart conversions.

\section{DISCUSSION}

While there were similarities in some aspects of the EHR implementation across the two types of small practices, there were some differences between IPPs and APPs. This section provides the implications of the study and highlights some best-practices for SMPPs in general (Table 4) based on overall findings of the study.

TABLE 4

Comparison Between the Affiliated Physician Practices (APP) and the Independent Physician Practice (IPP) for Adoption of Electronic Health Record (EHR) Use

\begin{tabular}{|c|c|c|c|c|}
\hline Practice Type & ERP Choice & $\begin{array}{l}\text { Common } \\
\text { Language }\end{array}$ & Rollout Type & Best Practices \\
\hline APPs & $\begin{array}{l}\text { Chosen by } \\
\text { parent } \\
\text { organization }\end{array}$ & $\begin{array}{l}\text { Not well versed } \\
\text { in EHR } \\
\text { terminology }\end{array}$ & $\begin{array}{l}\text { Single } \\
\text { switch-over } \\
\text { to new } \\
\text { system }\end{array}$ & $\begin{array}{l}\text { - Even without the choice of EHR, } \\
\text { practices can make the new system fit } \\
\text { their organization through } \\
\text { customization } \\
\text { - Provide more training at convenient } \\
\text { times }\end{array}$ \\
\hline IPP & $\begin{array}{l}\text { Choice based on } \\
\text { practice } \\
\text { requirements }\end{array}$ & $\begin{array}{l}\text { More familiar } \\
\text { with EHR } \\
\text { terminology }\end{array}$ & $\begin{array}{l}\text { Staggered } \\
\text { rollout } \\
\text { based on } \\
\text { physicians }\end{array}$ & $\begin{array}{l}\text { - Educate users on system benefits and } \\
\text { not just its use } \\
\text { - If possible, stagger the rollout to } \\
\text { alleviate disruptions to entire practice } \\
\text { - Use a champion to encourage use of } \\
\text { the new system }\end{array}$ \\
\hline
\end{tabular}

\section{Training}

It is vital that there is sufficient training prior to go-live date for a new system. While it takes actually using and working with the EHR to become comfortable with it, there must be enough time allowed to process the information taught and at least begin to personalize how the EHR works in the training. The most efficient means of training is to work through mock patient examinations with the new system. It is also recommended that the users are comfortable with the basic features of a new system before introducing the more advanced features. This approach serves to increase their self-efficacy and become more comfortable with using an unfamiliar interface. 
It is also critical to make sure that everyone understands the importance of the training and to motivate users to work hard during the training sessions. One study participant specifically said that he "put pressure on staff to be ready." This is also part of the culture change within the practice. In a SMPP, everyone will likely have a role during the implementation process and this will ensure that they will have a stake in a successful implementation.

Training cannot stop with the initial implementation of the system. There must be ongoing training that is available during a time when those attending are mentally fresh and alert. One subject stated that holding the training in the evening after a full day of work is not effective. One suggestion is to set aside a half of a workday every 2 to 4 weeks until the users are full proficient. If possible, one-on-one training will ensure that the trainee will receive personalized help with the system and make it easier to learn the more advanced feature that pertain to their role within the practice. Some EHR systems provide analytics that can show where more training is needed and, in some cases, even target specific deficiencies for particular users. If this feature is not provided, then data should be collected from the users to find out their training needs. We also suggest that this topic should be a line item during regularly held staff meetings to provide users an opportunity to discuss system issues and provide solutions for the entire staff.

\section{System Customization}

When possible, it is advisable to bring in outside help for training and possible customization. Practice 2 contracted an on-site trainer to be present prior to implementation. This gave the practice an opportunity to customize the patient charts, which made their workflow more efficient. Not all practices will have workflows that conform to a standardized EHR system, and changes to the system to fit the current workflow are preferable to changing the practice to fit the new system (Lee, Cain Young, Chockley, \& Burstin, 2005). Someone who has used the EHR for a while and is comfortable with the advanced features can offer great insights. Bringing in someone not affiliated with the vendor will also provide a different perspective.

\section{Importance of a Champion}

It is very interesting that the physician in Practice 2 was excited about the change to the new EHR system and had embraced it. Because of that enthusiasm, the physician was able to encourage the staff and to sell the benefits of EHR to the entire office. It was apparent during the interview that the physician was excited about the new system and felt compelled to talk about it. This was far different from the other cases in which no enthusiasm was evident with regards to the new EHR system. This point may be the difference between the organizational culture of a solo practice and a multiple physician practice. The solo practice may have the advantage of having a single physician who is in charge of the entire office and thus has the benefit of having more influence on the users. The other APPs may not have had that advantage and lacked a champion to market the system to the rest of the office. Another explanation can be found in the equity implementation model (Joshi,2005). Practice 2 may have also seen this new system favorably because they perceived the system to be equitable. Because the system did not hamper the performance of the physician, the new system was accepted. 
Practice 5 was another case that used a champion to help implement the system. In order to gain acceptance, the practice administrator enlisted one of the physicians to be the champion of the project, which involved becoming the first physician to go live in the EHR system. They were careful not to pick someone who is a "techie" so as not to initiate a system with an individual who might be positively biased toward technology. Instead the administrator picked someone who was not familiar with IT. This physician is described as someone "young and easy going" and who had prior experience with EMR during residency. As the project champion, this physician attended all of the meetings involved with the EMR implementation and helped with the implementation decisions, which included system customization, template creation, which documents to scan for the system, and which MU criteria to address. One week following this physician going live in the system, a second physician insisted on changing over to the new system. All eight physicians were live in the new system after 2 months.

As stated previously, SMPPs are ill equipped to deal with the lost revenue that incurred during implementation due to lost productivity. In the case of the APPs, they were able to overcome that obstacle due to the support given by their parent organization. Practice 5, an IPP, was able to offset the loss in their revenue by staggering the implementation within the practice and only disrupting one or two physicians at a time. Because SMPPs normally do not have their own IT staff due to size and budget, they have to rely on outside IT support and exploit that support as much as possible. Practice 2 took full advantage of their vendor's support by taking as many training classes as possible and requesting some customization when it would help their workflows. Some challenges are unique to SMPPs due to their size and resources, but these challenges can be overcome if proper planning has been performed.

\section{CONCLUSION}

Undertaking an EHR implementation is an immense task and requires a large investment in both time and money (Columbus,2006). However, with careful planning, enough time, and a dedicated staff, use of EHR can position a practice to provide better care more efficiently. This study through interviews with key informants provides some recommendations for a successful EHR implementation. We have shown that, by having a champion within a practice, EHR implementation can be executed with fewer challenges. We were also able to show that training can help prepare users prior to implementation. Finally, we were able to provide additional support for Lee et al. (2005) suggestion for EHR customization.

One of the limitations of this study is that we were only able to examine a single IPP (Practice 5). The other practices in our study were not responsible for the entire EHR system implementation process. In this study, the APPs had no role in the selection of the EHR system and thus had no voice in the many decisions required for implementation. Practice 5 did not have to shoulder all of the expenses for their new EHR system as the local hospital helped subsidize the implementation. This influenced their decision to implement a new EHR. By examining more IPPs, especially an IPP without outside financial assistance, we can further our understanding of SMPPs and their ability to successfully implement an EHR system.

Another limitation is that only one solo practice participated in this study (Practice 2) and thus another solo practice was lacking for comparison with Practice 2. It would have been beneficial to examine other solo practices for comparison and to discover if other solo practices have 
similar experiences. For future research, it would also be beneficial to study if general computer efficacy for the practice staff has any impact on the success of EHR system implementation in SMPPs.

\section{Notes}

2. ${ }^{1}$ Sandbox mode is a training version of the software application that only contains dummy data.

\section{REFERENCES}

1. Albert, C., Wallace, S., \& Iyer, L. S. (2011). Closing the gaps in quality health care: An exploratory case study of health care information systems usage in independent medical practices. Proceedings of the Annual Meeting of the Global Information Technology Management Conference, Las Vegas, NV.

2. Bardach N. S., Wang J. J., De Leon S. F., Shih, S. C., Boscardin, W. J., Goldman, L. E., \& Dudley R. A. (2013). Effect of pay-for-performance incentives on quality of care in small practices with electronic health records: A randomized trial. Journal of the American Medical Association, 310(10), 1051-1059.

3. Baron R., Fabens E., Schiffman, M., \& Wolf, E. (2005). Electronic health records: Just around the corner? Or over the cliff?Annals of Internal Medicine, 143(3), 222-226.

4. Beasley, J. W., Ben-Tzion, K., Hagenauer, M. E., Marchand, L., \& Sainfort, F. (2005). Quality of work life of independent vs employed family physicians in Wisconsin: A WReN study. Annals of Family Medicine, 3(6), 500-507.

5. Bentley, T. G. K., Effros, R. M., Palar, K., \& Keeler, E. B. (2008). Waste in the U.S. health care system: A conceptual framework.Milbank Quarterly, 86(4), 629-659.

6. Berry, L. L., Seiders, K., \& Wilder, S. S. (2003). Improving patient care innovations in access to care: A patient-centered approach. Annals of Internal Medicine, 139, 568-574.

7. Berwick, D. M. (2002). A user's manual for the IOM's "quality chasm" report. Health Affairs, 21(3), 80-90.

8. Carayon, P., Smith, P., Hundt, A. S., Kuruchittham, V., \& Li, Q. (2009). Implementation of an electronic health records system in a small clinic: The viewpoint of clinic staff. Behaviour \& Information Technology, 28(1), 5-20.

9. Cecchini, S. L. (2011a). CHISP certification review and study guide. Salt Lake City, UT: American Society of Health Informatics Managers.

10. Cecchini, S. L. (2011b). Health IT professional training program. Salt Lake City, UT: American Society of Health Informatics Managers. 
11. Centers for Medicare \& Medicaid Services (CMS). (2014). Centers for Medicare and Medicaid-State breakdown of payments to Medicare and Medicaid providers through January 31, 2014. Retrieved from http://www.cms.gov/Regulations-andGuidance/Legislation/EHRIncentivePrograms/DataAndReports.html

12. Columbus, S. (2006). Small practice, big decision: Selecting an EHR system for small physician practices. Journal of AHIMA,77(5), 42-46.

13. Couchman, G. R., Forjuoh, S. N., \& Rascoe, T. G. (2001). E-mail communications in family practice: What do patients expect?Journal of Family Practice, 50(5), 414-418.

14. Davidson, E., \& Heslinga, D. (2007). Bridging the IT adoption gap for small physician practices: An action study on electronic health records. Information Systems Management, 24(1), $15-28$.

15. Dey A., Sinha K. K., \& Thirumalai, S. (2013). IT capability for health care delivery: Is more better? Journal of Service Research,16(3), 326-340.

16. Downing Peck, A. (2013). EHR implementation: Training pays dividends. Retrieved from http://medicaleconomics.modernmedicine.com/medical-economics/RC/ehr-implementation$\underline{\text { training-pays-dividends?page }=\text { full }}$

17. Eastaugh, S. R. (2012). Health information technology impact on productivity. Journal of Health Care Finance, 39(2), 64-81.

18. Edwards, P. J. (2008). Maximizing your investment in EHR: Utilizing EHRs to inform continuous quality improvement. Journal of Healthcare Information Management, 22(1), 3237.

19. Fleming, N. S., Aponte, P., Ballard, D. J., Becker, E., Collinsworth, A., Culler, S., ... Chang, D. (2011). Exploring financial and non-financial costs and benefits of health information technology: The impact of an ambulatory electronic health record on financial and workflow in primary care practices and costs of implementation. Grant Report for AHRQ. Grant ID R03HS018220. Retrieved from http://healthit.ahrq.gov/sites/default/files/docs/publication/R03HS018220$\underline{\text { 01Flemingfinalreport2011.pdf }}$

20. Furumo, K., \& Melcher, A. (2006). The importance of social structure in implementing ERP systems: A case study using adaptive structuration theory. Journal of Information Technology Case and Application Research, 8(2), 39-58.

21. Gaal, S., Laarhoven, E., Wolters, R., Wetzels, R., Verstappen, W., \& Wensing, M. (2010). Patient safety in primary care has many aspects: An interview study in primary care doctors and nurses. Journal of Evaluation in Clinical Practice, 16, 639-643. 
22. Gans, D., Kralewski, J., Hammons, T., \& Dowd, B. (2005). Medical groups' adoption of electronic health records and information systems. Health Affairs, 24(5), 1323-1333.

23. Garret, P., \& Seidman, J. (2011). HealthIT.gov-EMR vs EHR: What is the difference? Retrieved fromhttp://www.healthit.gov/buzz-blog/electronic-health-and-medicalrecords/emr-vs-ehr-difference/

24. HealthIT. (2013). HealthIT.gov-How to implement EHRs. Retrieved from http://www.healthit.gov/providers-professionals/ehr-implementation-steps

25. Health Resources and Services Administration (HRSA). (2013). Health Resources and Services Administration-What is meaningful use? Retrieved from http://www.hrsa.gov/healthit/meaningfuluse/MU\%20Stage1\%20CQM/mu.html

26. Henricks, W. H. (2011). "Meaningful use" of electronic health records and its relevance to laboratories and pathologists. Journal of Pathology Informatics, 2(7), 1-14.

27. Hoffmann, L. (2009). Implementing electronic medical records. Communications of the ACM, 52(11), 18-19.

28. Institute of Medicine (IOM). (2001). Institute of Medicine (US) - Crossing the quality chasm: A new health system for the 21st century., Committee on Quality of Health Care in America. Washington, DC: National Academies Press.

29. Isaacs, S., Jellinek, P., \& Ray, W. (2009). The independent physician-Going, going... New England Journal of Medicine, 360(7), 655-657.

30. Joshi, K. (2005). Understanding user resistance and acceptance during the implementation of an order management system: A case study using the equity implementation model. Journal of Information Technology Case and Application Research,7(1), 6-20.

31. Kealy, A., \& Stapleton, L. (2011). Symbols of hope: A case study of telemedicine projects in post conflict regions. Journal of Information Technology Case and Application Research, 14(3), 3-32.

32. Klein, H. K., \& Myers, M. D. (1999). A set of principles for conducting and evaluating interpretive field studies in information systems. MIS Quarterly, 23(1), 67-93.

33. Koh, C., Soh, C., \& Markus, M. L. (2000). A process theory approach to analyzing ERP implementation and impacts: The case of Revel Asia. Journal of Information Technology Case and Application Research, 2(1), 4-23.

34. Lee J., Cain C., Young S., Chockley N., \& Burstin H. (2005). The adoption gap: Health information technology in small physician practices. Health Affairs, 24(5), 1364-1367. 
35. Lorenzi N. M., Kouroubali A., Detmer D. E., \& Bloomrosen M. (2009). How to successfully select and implement electronic health records (EHR) in small ambulatory practice settings. BMC Medical Informatics and Decision Making, 9(1), 1-13.

36. Ludwick D. A., \& Doucette J. (2009). Adopting electronic medical records in primary care: Lessons learned from health information systems implementation experience in seven countries. International Journal of Medical Informatics, 78(1), 22-31.

37. Manos, D. (2013). EHR's not enough, study finds. Retrieved from http://www.healthcareitnews.com/news/ehrs-not-enough-study-finds

38. Markus, M. L., \& Tanis, C. (2000). The enterprise systems experience-From adoption to success. In Zmud, R. W. (Ed.), Framing the domains of IT Research: Projecting the future ... through the past (pp. 173-207). Cincinnati, OH: Pinnaflex Education Resources, Inc.

39. National Learning Consortium (NLC). (2012a). The National Learning ConsortiumElectronic health record (EHR) system testing plan. Retrieved from http://www.healthit.gov/providers-professionals/implementation-resources/electronichealth-record-ehr-system-testing-plan

40. National Learning Consortium (NLC). (2012b). The National Learning ConsortiumWorkflow process mapping for electronic health record (EHR) implementation. Retrieved from http://www.healthit.gov/providers-professionals/implementation-resources/workflowprocess-mapping-electronic-health-record

41. O’Neill, L., Talbert, J., \& Klepack, W. (2011). Physician characteristics associated with early adoption of electronic medical records in smaller group practices. In J. Tan (Ed.), New technologies for advancing healthcare and clinical practices (pp. 182-191). Hershey, PA: IGI Global.

42. Otieno, G. O., Hinako, T., Motohiro, A., Daisuke, K., \& Keiko, N. (2008). Measuring effectiveness of electronic medical records systems: Towards building a composite index for benchmarking hospitals. International Journal of Medical Informatics,77(10), 657-69.

43. Palvia, P., \& Thambusamy, R. (2013). Electronic medical records application development: Perspectives of the service provider. Journal of Information Technology Case and Application Research, 15(2), 11-29.

44. Pedersen, K., Kraemmergaard, P., Lynge, B. C., \& Schou, C. D. (2010). ITIL implementation: Critical success factors a comparative case study using the BPC framework. Journal of Information Technology Case and Application Research, 12(2), 11-35. 45. Reardon J. L., \& Davidson E. (2007). An organizational learning perspective on the assimilation of electronic medical records among small physician practices. European Journal of Information Systems, 16(6), 681-694. 
46. Reardon, J. L. (2011). Perceptions of an organizing vision for electronic medical records by independent physician practices. In Tan, J. (Ed.), New technologies for advancing healthcare and clinical practices (pp. 211-233). Hershey, PA: IGI Global.

47. Robinson, J., Callister, L., Berry, J., \& Dearing, K. (2008). Patient-centered care and adherence: Definitions and applications to improve outcomes. Journal of the American Academy of Nurse Practitioners, 20, 600-607.

48. Terry, K. (2013). Doctors are increasingly dissatisfied with EHRs. Retrieved from http://www.ihealthbeat.org/insight/2013/doctors-are-increasingly-dissatisfied-with-ehrs 49. Thompson, S. M., \& Dean, M. D. (2009). Advancing information technology in health care. Communications of the ACM, 52(6), 118.

50. Tollen L. A. (2008). Physician organization in relation to quality and efficiency of care: A synthesis of recent literature.Commonwealth Fund, 2008 (April), 1-30.

51. Venkatraman S., Bala H., Venkatesh, V., \& Bates, J. (2008). Six strategies for electronic medical records systems.Communications of the ACM, 51(11), 140-144.

52. Walsham, G. (2006). Doing interpretive research. European Journal of Information Systems, 15(3), 320-330.

53. Ward, R., Stevens, C., Brentnall, P., \& Briddon, J. (2008). The attitudes of health care staff to information technology: A comprehensive review of the research literature. Health Information and Libraries Journal, 25(2), 81-97.

54. Wietecha, G. M. (2013). EHR replacement-Do it right. Retrieved from http://www.healthcareitnews.com/resource/ehr-replacement-\%E2\%80\%93-do-it-right

55. Williams F. \& Boren S. A. (2008). The role of electronic medical record in care delivery in developing countries. International Journal of Information Management, 28(6), 503-507.

56. Yin, R. K. (2009). Case study research: Design and methods. Washington, DC: Sage. 